\title{
ARTICLE
}

\section{Neurocognitive problems in epilepsy}

\author{
Shruti Lodhi \& Niruj Agrawal
}

\begin{abstract}
Shruti Lodhi is an ST6 in old age psychiatry in South West London and St George's Mental Health NHS Trust. She has a particular interest in the neuropsychiatric consequences of memory disorders. Niruj Agrawal is a consultant neuropsychiatrist and honorary senior lecturer providing tertiary neuropsychiatry services to the Atkinson Morley Regional Neurosciences Centre at St George's Hospital, London and the Wolfson Neurorehabilitation Centre in Wimbledon. He has particular interest in the neuropsychiatric consequences of epilepsy, acquired brain injuries and memory disorders Correspondence Dr Niruj Agrawal, Consultant Neuropsychiatrist, Department of Neuropsychiatry, Clare House, St George's Hospital, Blackshaw Road, London SW17 00T, UK. Email: Niruj. Agrawal@swlstg-tr.nhs.uk
\end{abstract}

\begin{abstract}
SUMMARY
Cognitive dysfunction is one of the major contributors to the burden of epilepsy. It can significantly disrupt intellectual development in children and functional status and quality of life in adults. Epilepsy affects cognition through a number of mechanisms in complex interrelationship. Cognitive deficits in epilepsy may be treated indirectly through aggressive seizure control using anti-epileptic drugs or surgery, and by treating comorbid conditions such as depression. The beneficial effects of reducing seizures may offset the adverse cognitive side-effects of these therapies. Direct treatment of cognitive impairment in epilepsy mainly involves memory rehabilitation. Other direct treatments are mostly experimental and their evidence base is currently poor.

\section{DECLARATION OF INTEREST}

None.
\end{abstract}

The consequences of epilepsy are often as serious as having the disorder itself. One particularly important consequence is the impairment of cognitive function. The prevalence of memory disorders in people with epilepsy is between 20 and $50 \%$, and over half of these individuals report memory difficulties in daily life. Some patients find these cognitive consequences more debilitating than the actual seizures (Aldenkamp 2006).

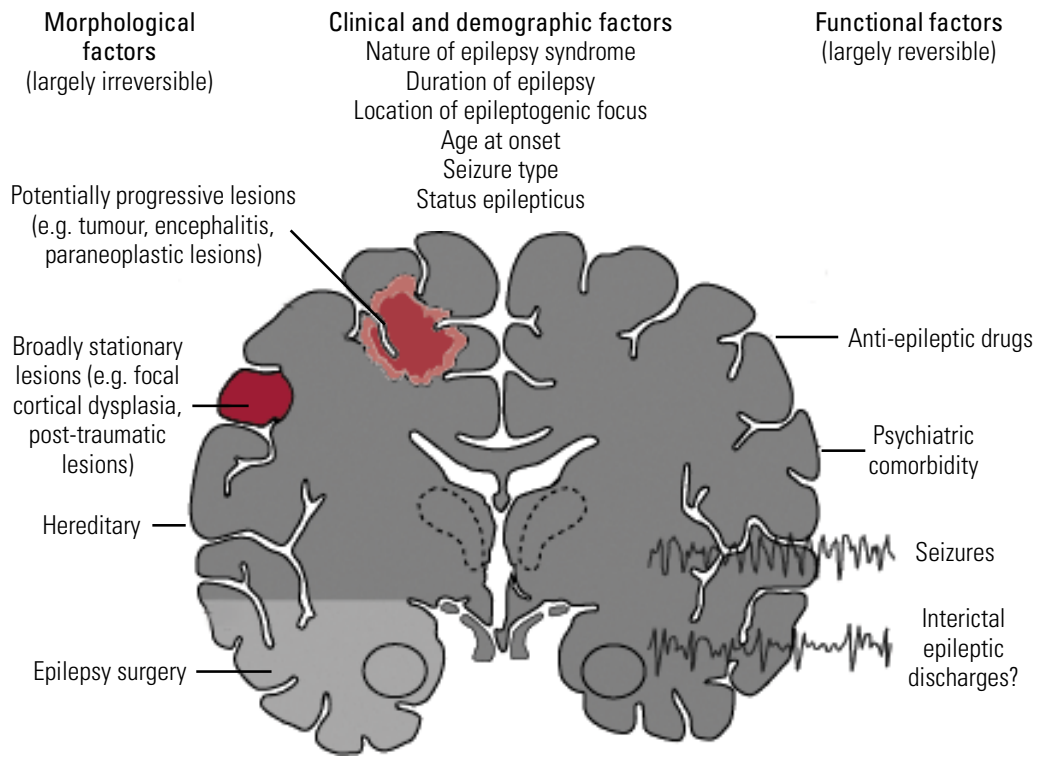

Cognitive function is defined as higher brain function, that is, the capacity of the brain to programme adaptive behaviour, solve problems, memorise information and focus attention. Epilepsy is a chronic neurological condition characterised by recurrent unprovoked seizures. Cognitive impairment often develops as a symptom secondary to the disorder. The most frequently reported cognitive problems associated with epilepsy are memory impairment, mental slowing and attentional deficits. These can have a significant impact on a number of day-today functions, such as school work in children and driving ability in adults. Epilepsy can also further impair already compromised cognitive function, for example in elderly people. It is therefore no surprise that people with epilepsy often report a poor quality of life resulting from the complex interactions between the physical and psychosocial factors related to their condition (Aldenkamp 2006).

\section{Factors affecting cognition in epilepsy}

A number of factors influence the behavioural and cognitive impact of epilepsy (Fig. 1). These include the aetiology of the disorder (in particular, whether there is underlying brain damage), epilepsy-related factors (e.g. the nature of the epilepsy syndrome, duration of illness, location of the epileptogenic focus, age at onset), seizure-related factors (e.g. seizure type, seizure frequency, occurrence of status epilepticus) and treatment-related factors (e.g. type of anti-epileptic drug, dosage, drug interactions). This review discusses recent progress in our understanding of these factors. Box 1 gives a glossary of key terms and Box 2 a list of the abbreviations most commonly used in the literature.

\section{Seizure aetiology}

Where the aetiology of seizures involves underlying brain damage it may be relevant to cognitive dysfunction. Cortical dysplasia is a congenital abnormality mainly found near the cerebral cortex, and it is often associated with seizures. In epilepsies associated with cortical dysplasia, the extent of the underlying malformation is an important determinant of intellectual impairment. Structural brain damage that produces cognitive impairment may also appear as a long-term 


\section{B0X 1 Glossary}

Absence seizures Brief (usually less than 20 s) generalised epileptic seizures of sudden onset and termination. They have two essential components: the impairment of consciousness (absence); and electroencephalograph (EEG) generalised spike-and-slowwave discharges.

Cryptogenic epilepsy Involves a presumptive lesion that is otherwise difficult or impossible to uncover during evaluation.

Generalised epilepsy The source of the seizure within the brain is distributed. Seizures are divided according to the effect on the body, but all involve loss of consciousness. They include absence (petit mal), myoclonic, clonic, tonic, tonic-clonic (grand $\mathrm{mal}$ ) and atonic seizures.

Idiopathic epilepsies Thought to arise from genetic abnormalities that lead to alteration of basic neuronal regulation.

Myoclonic seizures Can be described as 'jumps'. They are caused by rapid contraction and relaxation of the muscles.

Partial (focal) epilepsy The source of the seizure within the brain is localised. Partial seizures are further divided depending on the extent to which consciousness is affected. If it is unaffected, then it is a simple partial seizure; otherwise, it is a complex partial (psychomotor) seizure. A partial seizure may spread within the brain, a process known as secondary generalisation.

Status epilepticus A life-threatening condition, which is always considered a medical emergency, where the brain is in a state of persistent seizure. It is defined as one continuous unremitting seizure lasting longer than $5 \mathrm{~min}$, or recurrent seizures without regaining consciousness between seizures for longer than $5 \mathrm{~min}$.

Symptomatic epilepsies Arise from the effects of an epileptic lesion, whether that lesion is focal, such as a tumour, or a defect in metabolism causing widespread injury to the brain.

Tonic-clonic seizures A type of generalised seizure that affects the entire brain (formerly known as grand mal seizures). The seizures are divided into two phases, the tonic phase and the clonic phase, and are often preceded by an aura.

consequence of uncontrolled epilepsy, for example hippocampal sclerosis in temporal lobe epilepsy (Aldenkamp 2005).

\section{Duration of seizure}

There is a broad relationship between the type of seizure and cognitive impairment. Absence seizures in children (Box 1) tend to be associated with less cognitive impairment than convulsive seizures. Generalised tonic-clonic seizures are associated with greater cognitive impairment than partial (focal) seizures; and the highest risk is observed with status epilepticus (Lezak 2004).

The study of the idiopathic epilepsies may be helpful in understanding the relationship between cognitive impairment and the stable, disease-related characteristics of epilepsy and its paroxysmal epileptic activity. It also provides a study model for the extent and limits of cerebral plasticity, as well as for the evaluation of the effects of epilepsy on development and learning.

\section{Nature of epilepsy syndrome}

\section{Idiopathic generalised epilepsies}

Idiopathic generalised epilepsies are characterised by seizures that are generalised and associated with bilateral, synchronous and symmetrical electroencephalograph (EEG) spikes and waves, or polyspike waves. They comprise benign myoclonic epilepsy in infancy, childhood absence epilepsy, juvenile absence epilepsy, juvenile myoclonic epilepsy, and epilepsy with generalised tonicclonic seizures (Hommet 2006).

\section{Juvenile myoclonic epilepsy}

The most common form of idiopathic generalised epilepsy is juvenile myoclonic epilepsy, also known as Janz syndrome. The first manifestations occur between the ages of 12 and 18, with myoclonus early in the morning. Most patients also have tonic-clonic seizures and many also have absence seizures (Nordli 2005).

Research implicates frontal lobe dysfunction in juvenile myoclonic epilepsy. Neuropsychological studies have shown impaired executive functions,

BOX 2 Common abbreviations and alternative nomenclatures

BCECTS benign childhood epilepsy with centrotemporal spikes (benign Rolandic epilepsy)

CAE childhood absence epilepsy

FLE frontal lobe epilepsy

IGE idiopathic generalised epilepsy

IPE idiopathic partial epilepsy

JME juvenile myoclonic epilepsy

LGS Lennox-Gastaut syndrome (childhood epileptic encephalopathy)

LKS Landau-Kleffner syndrome (infantile acquired aphasia, acquired epileptic aphasia, aphasia with convulsive disorder)

TEA transient epileptic amnesia

TLE temporal lobe epilepsy

VNS vagal nerve stimulation 
involving concept formation, abstract reasoning, mental flexibility, cognitive speed and planning. Neuropathological findings have shown microdysgenetic lesions (microscopic abnormalities in cytoarchitecture) in the neocortex and subcortical white matter of the frontal lobes and hippocampus. Magnetic resonance (MR) spectroscopy reveals reduced $N$-acetylaspartate concentrations in the prefrontal cortex, whereas ${ }^{18} \mathrm{FDG}$ positron emission tomography (PET) shows reduced glucose metabolism in the prefrontal cortex during mental tasks (Hommet 2006).

\section{Idiopathic partial epilepsies}

Idiopathic partial (focal) epilepsies are agedependent syndromes that occur in the absence of a significant anatomical lesion, even though they have a localised origin. The clinical examination is usually normal, and the seizures disappear spontaneously during adolescence.

\section{Benign childhood epilepsy with centrotemporal spikes}

The most frequently studied idiopathic partial epilepsy is benign childhood epilepsy with centrotemporal spikes, also known as benign Rolandic epilepsy (Hommet 2006). This syndrome provides a good model for the effect of epilepsy on cognition, because there are no confounding variables from additional cerebral diseases (Elger 2004). Most children outgrow this epilepsy by their mid to late teens, hence the label benign (even though it can cause deficits).

The seizures start around the central sulcus of the brain. They are characterised by either simple partial seizures involving the mouth and face, or generalised tonic-clonic seizures. The topography of the epileptic discharges on EEG traces has aroused interest in the study of language functions in this form of epilepsy. Deficits have been found in reading, writing, morphosyntax, auditory-verbal learning and auditory discrimination. Other deficits include impairments in visual and perceptive abilities, short-term memory, fine motor control, executive functions and attention (Hommet 2006).

\section{Acquired epileptic aphasia}

The study of specific epileptic syndromes such as acquired epileptic aphasia (Landau-Kleffner syndrome) provides clinical evidence for the impact of epileptiform EEG discharges on cognitive function. Acquired epileptic aphasia typically occurs between the 3 and 8 years of age, which coincides with a phase of accelerated cerebral maturation. The prolonged exposure to abnormal neural activity during this critical period leads to changes in language development, learning and lateralisation of functions. Receptive speech is lost first, then expressive speech deteriorates. Although the condition remits in adolescence, severe language impairment may persist (Hommet 2006). These deficits point to permanent structural damage in the auditory-verbal system.

\section{Symptomatic and cryptogenic epilepsies}

Most symptomatic and cryptogenic epilepsies are clearly focal in character, but some severe childhood epilepsies, such as childhood epileptic encephalopathy (Lennox-Gastaut syndrome), are multifocal or 'pseudo-generalised'. They are characterised by frequent seizures of various types and are often accompanied by intellectual disability and behavioural problems (Elger 2004).

\section{Location of the epileptogenic focus}

The localisation of the epileptic focus is an important determinant of the extent and nature of cognitive deficits. Patients with focal seizure onset display a pattern of test performance like that of patients with non-epileptogenic lesions in similar places. However, the magnitude of the cognitive deficits is often less than with comparable nonepileptogenic lesions. Left-hemisphere seizures are associated with impaired verbal functions. Righthemisphere seizures display visuoperceptual, visual memory and constructional disabilities (Lezak 2004).

\section{Temporal lobe epilepsy}

Temporal lobe epilepsy is associated with particular memory problems: verbal memory deficits in left-temporal lobe epilepsy and visual memory deficits in right-temporal lobe epilepsy (Elger 2004). Other deficits in this symptomatic focal epilepsy include long-term memory decline due to disturbance of memory consolidation. This may be caused by anatomical cortical disruption ('structural disconnection') resulting from the epileptic seizures themselves, and/ or by the impairment of neurochemical and neurophysiological processes. Refractory temporal lobe epilepsy is associated with ongoing cognitive deterioration. This is thought to be due to the accumulation of small neurodegenerative effects of noxious neurochemical agents, abnormal brain electrical events and metabolic disturbances that exhaust the compensatory capacity of brain functions over decades of seizures. Long-term memory impairment adversely affects learning, working abilities and social status, and is therefore of paramount importance to people with epilepsy (Mameniskiene 2006). 


\section{Transient epileptic amnesia}

A subtype of temporal lobe epilepsy that it is important to consider when studying the impact of epilepsy on cognition is transient epileptic amnesia. The main manifestation of the seizure is amnesia, which usually lasts for less than an hour. It can also be associated with interictal complaints of accelerated forgetting and autobiographical amnesia (Butler 2009).

Accelerated forgetting refers to the excessively rapid loss of newly acquired memories over a period of days or weeks. The phenomenon suggests an impairment of memory consolidation processes. Although no single reason has been identified to explain this problem, several possible causes have been suggested (Zeman 1998; Blake 2000; Butler 2007, 2008):

- seizure activity: abnormal electrical activity during sleep may disrupt the process of memory consolidation that normally occurs in sleep;

- brain pathology: damage to the temporal lobes may both cause seizures and interfere with the handling of memories;

- anticonvulsant medication: this is an unlikely cause, given that the memory problems tend to exist before the initiation of medication and often improve following treatment;

- psychological mechanisms: low mood and low self-esteem do not play an important role in transient epileptic amnesia.

Autobiographical amnesia is a neurogenic retrograde amnesia that involves the loss of memories of salient personal events experienced in previous decades.

\section{Frontal lobe epilepsy}

Few neuropsychological studies have attempted to assess the behavioural characteristics and nature of impairment in frontal lobe epilepsy (McCagh 2009). However, there is some evidence that the cognitive dysfunction in this form of epilepsy is similar to that in other frontal lobe diseases or lesions. People with frontal lobe epilepsy have deficits in motor skills, response inhibition, attention, working memory, planning and psychomotor speed (Elger 2004). Hence, it appears that frontal lobe epilepsy gives rise to deficits in executive functioning.

\section{Seizure frequency}

Repeated seizures are associated with increased intellectual impairment. A negative correlation between frequency of generalised tonic-clonic seizures and performance on neuropsychological and IQ tests has been observed (Fig. 2) (Aldenkamp 2005).

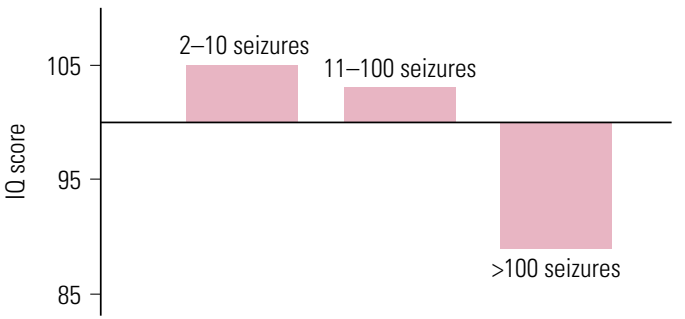

I0 score as a function of seizure frequency (lifetime number of attacks) in 94 adults with secondary generalised tonic-clonic seizures (Dodrill 1986, permission requested).

The relationship between cognitive deterioration and seizure frequency was examined in a study involving 24 patients with secondary generalised seizures of unknown aetiology. They were divided into two groups according to lifetime seizure frequency: over 50 seizures and under 10 seizures. Those who had experienced fewer than 10 seizures had a normal IQ, whereas those who had had more than 50 seizures showed a lower IQ than agematched population norms (Aldenkamp 2005).

Functional magnetic resonance imaging (fMRI) in healthy individuals and those with epilepsy of various seizure frequency has revealed differences in the pattern of brain activation during the Sternberg Item Recognition Paradigm. In the healthy individuals and those with a lifetime seizure frequency of under 10, fMRI showed similar activation in specific areas, i.e. the dorsolateral frontal cortex, anterior cingulate cortex (executive function) and posterior parietal cortex (attention). Participants with a lifetime seizure frequency of over 50, however, revealed a disseminated pattern of brain activation, rather than the focused pattern of the other two groups (Aldenkamp 2005).

As well as the accrual of intellectual deterioration due to multiple seizures, individual generalised tonic-clonic seizures produce reversible attentional slowing. A single seizure can generate attentional slowing in the post-ictal phase that continues for at least 24 hours (Fig 3; Aldenkamp 2005).

\section{Duration of illness}

Prolonged refractory temporal lobe epilepsy may cause a deterioration of general intellectual functioning. However, the cognitive decline progresses very slowly and may be a result of normal ageing, as in people without seizures (Elger 2004).

\section{Age at onset}

Age at onset appears to be a critical determinant of the cognitive and behavioural impact of epilepsy. There is a correlation between the level of risk of 


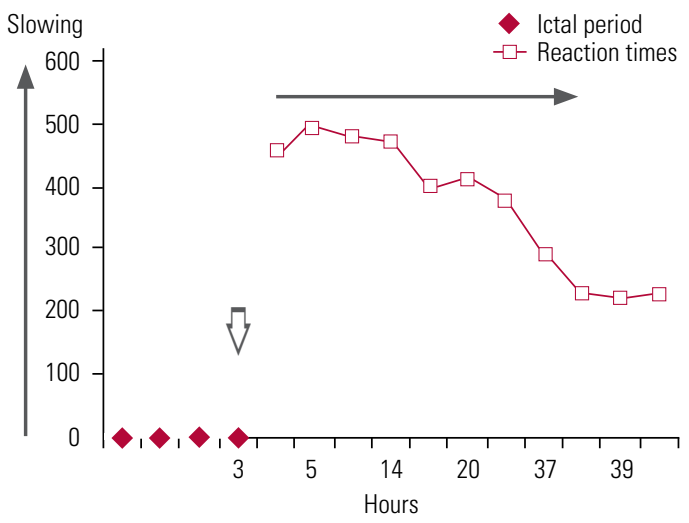

FIG 3

Post-ictal attentional deficit (slowing) after a single seizure in a patient with secondary generalised epilepsy (Aldenkamp 2005, permission requested).

decline in IQ and the age at which the child develops epilepsy: the younger the child at seizure onset, the higher the effect on their IQ. The onset of seizures before 5 years of age appears to be a particular risk factor for low IQ (Aldenkamp 2005). Acquired epileptic aphasia presents between the ages of 3 and 8 , the period in which the phase of accelerated cerebral maturation occurs.

\section{Anxiety and depression}

People with epilepsy are at greater risk of psychopathology, including anxiety, depression and suicide, than the general population. Even though psychiatric and psychological disorders are underdiagnosed in this cohort, depression and anxiety are 4-5 times more prevalent than in the general population (McCagh 2009).

Depression and anxiety are associated with a number of epilepsy-related factors, such as younger age at onset, longer duration of illness, poor seizure control, side-effects of anti-epileptics, increased seizure activity and greater seizure severity. They may also be a consequence of the unpredictability of epilepsy, restricted social activities, low self-esteem, stigma, and rejection by society (McCagh 2009).

Anxiety disorders are associated with episodic memory dysfunction, primarily related to acquisition rather than retrieval (Airaksinen 2005). Neuropsychological deficits in depression involve attention, memory, psychomotor speed, processing speed and executive function (Gualtieri 2006). The cognitive effects of anxiety and depression can exacerbate any cognitive dysfunction resulting from epilepsy itself.

\section{Anti-epileptic drugs}

Anti-epileptics decrease neuronal excitability, which may affect cognitive function. The severity of their cognitive side-effects is considered to be mild to moderate. However, their impact may be substantial if critical functions are involved, such as learning in children or driving capacity in adults, or if functions are impaired that are already vulnerable, such as memory in elderly people (Aldenkamp 2001a).

The neuropsychological functions adversely affected by anti-epileptics are psychomotor speed, vigilance, memory, mood and language, with disturbances such as dysphasia and anomia. Generally, patients give the newer anti-epileptics a more favourable cognitive profile than the older drugs.

The relatively new drug topiramate is an exception, as it is associated with particularly problematic cognitive, behavioural and psychiatric adverse events. Significant deterioration in verbal IQ, verbal fluency and verbal learning was recorded following introduction of topiramate as adjunctive therapy for people with intractable epilepsy (Aldenkamp 2005). Cognitive adverse effects of topiramate are dose-related: psychomotor slowing is observed at doses of $200 \mathrm{mg} /$ day, and language disturbances at $400 \mathrm{mg} /$ day or higher (Privitera 1996).

It has been postulated that the cognitive side-effects of topiramate and some other antiepileptics are related to their modulation of $\gamma$-aminobutyric acid (GABA) receptors, increasing the availability of GABA in the cerebral cortex. Increased GABAergic activity in the prefrontal cortex could lead to mental slowing, and its spread to the dorsolateral areas, including Broca's area, could underlie impairments of language production. Anti-epileptics such as lamotrigine do not primarily act on GABAergic transmission, and thus may be associated with lesser cognitive and behavioural impairments (Aldenkamp 2005). Lamotrigine is reported also to have antidepressant effects and has been associated with improvements in health-related quality of life factors (perceived cognitive abilities, dysphoria and tiredness) (Aldenkamp 2001b).

Cognitive adverse events are especially prominent in patients receiving anti-epileptic polytherapy, and a switch to monotherapy has been associated with an improvement in cognitive function (Brodie 1987).

Drugs that act on sodium channels are associated with the fewest cognitive side-effects, whereas the risk is greatest in those with GABAergic action. The sodium channel blockers lamotrigine and oxcarbazepine may have a positive effect on some cognitive functions, including learning and psychomotor speed. Anti-glutamatergic drugs (such as felbamate and lamotrigine) are associated more with positive effects on learning 
and memory, especially those that work on the $N$-methyl-D-aspartic acid (NMDA) receptor. Levetiracetam binds to a pre-synaptic protein SV2A, modulating neurotransmitter release. It showed an improvement in reaction times and memory for simultaneously presented words (Lagae 2006).

\section{Anti-epileptics in childhood epilepsy}

Children with epilepsy have a higher prevalence of cognitive and behavioural disturbances than their unaffected peers. It is imperative to identify the deficits promptly, as they can hinder neurological development and academic progress. Children with early-onset seizures, multiple seizure types, frequent seizures and/or severe epilepsy tend to underperform on IQ tests. It appears that the most important factor influencing improved cognition is becoming seizure-free (Hirsch 2003).

Older drugs, such as phenobarbital, phenytoin, carbamazepine and valproate, appear to induce psychomotor slowing. Although this may seem to be minimal, the increase in reaction time can be critical in everyday situations and for learning. In the past, phenobarbital was used as preventive medication for febrile seizures in children. However, its prescription for this purpose has now reduced as its long-term use has been associated with a decrease of up to 10 IQ points (Lagae 2006).

\section{Cognitive effects of anti-epileptics in elderly people}

Elderly patients are particularly vulnerable to the side-effects of anti-epileptics. However, as is the case with many drugs, the pharmacokinetics and pharmacodynamics of anti-epileptics alter in elderly people, so that patients respond to lower doses (Hirsch 2003).

General recommendations to improve adherence in this population include choosing broad-spectrum anti-epileptics with linear pharmacokinetics and fewer potential drug interactions, and twicedaily dosing. Potentially low-risk medications include valproic acid, lamotrigine, gabapentin and, possibly, levetiracetam, all of which have good pharmacokinetic profiles. When considering anti-epileptics, it is important to take into account comorbid psychiatric conditions, for example valproate is sedating, anxiolytic and antimanic, whereas lamotrigine is activating, anxiogenic and possibly antidepressant (Hirsh 2003).

\section{Epilepsy surgery}

Surgery is an established treatment for refractory epilepsy and it is more commonly used nowadays. Although group studies consistently demonstrate

post-operative deterioration in memory function, it is important to acknowledge that the majority of individual patients do not demonstrate such deterioration on standardised clinical memory tests. In fact, up to $25 \%$ demonstrate postoperative improvement in memory. The risks of surgery vary according to the characteristics of each patient (Langfitt 1996; Leijten 2005; Sanyal 2005). Pre-operative factors that affect postoperative memory are summarised in Box 3 .

\section{Prognosis of cognitive impairment}

Dementia resulting from progressive long-term cognitive impairment in pharmacoresistant epilepsy is a controversial concept. Helmstaedter $\&$ Elger (1999) have described it as a phantom. A plethora of evidence from cross-sectional and longitudinal studies shows that the progress of cognitive decline in chronic epilepsy is very slow and that, in adult epilepsies, successful treatment can stop or partly reverse it. In elderly people, any cognitive decline associated with epilepsy is usually due to early epilepsy-related deficit that then follows the course of physiological ageing (Elger 2004).

\section{Management of cognitive problems}

Cognitive deficits in epilepsy are usually managed indirectly by aggressive seizure control (including earlier epilepsy surgery), selecting anti-epileptics with good cognitive profiles, and treating comorbid conditions such as depression. The beneficial effects of anti-epileptics in reducing seizures may offset

BOX 3 Pre- and post-operative factors affecting neurocognition

- Pre-operative cognitive function Good pre-operative memory function is a risk for post-operative cognitive decline. In the simplest terms, patients who have 'lost' memory function stand to lose far less post-operatively than those who come to surgery with largely intact memory (Baxendale 2008)

- Age at time of surgery This appears to affect cortically represented learning and data acquisition skills more than consolidation and retrieval abilities

- Gender Women with left temporal lobe epilepsy tend to recall more words than men on a verbal learning task both before and after surgery regardless of the extent of hippocampal damage; these differences may be due to the different coding strategies used by men and women
- Plasticity Functional MRI studies in people with temporal lobe epilepsy and those who have had epilepsy surgery show verbal memory shift to the language non-dominant hemisphere in left temporal lobe epilepsy even in the absence of a corresponding transfer of language functions (Gleissner 2002; Helmstaedter 2004)

- Number of seizures Reduction or cessation of anti-epileptics following surgery leads to improvements in memory, speed of processing and language skills (Baxendale 2008)

- Laterality Verbal memory deficits are certainly more common following a dominant temporal lobe resection. A selective amygdala-hippocampectomy may be associated with fewer postoperative cognitive changes than a standardised en bloc temporal resection 
MCO answers

$1 d \quad 2$ e $\quad 3 d \quad 4 c \quad 5 a$ their adverse cognitive effects. Direct therapy for cognitive deficits associated with epilepsy, such as vagal nerve stimulation, cholinergic replacement and stimulants have little evidence base, and are therefore not recommended in clinical practice. Memory rehabilitation strategies could be more helpful as a direct treatment for significant cognitive difficulties.

\section{Vagal nerve stimulation}

Vagal nerve stimulation is a licensed treatment for refractory epilepsy that can also improve depression and memory problems. Both of these effects could help reduce cognitive impairment in epilepsy. Stimulation of the vagus nerve improves word recognition in people with epilepsy. Intermittent, moderate-intensity stimulation is the most effective: chronic and high-intensity stimulation appear not to improve cognitive functioning (Shulman 2002). Further systematic data collection from routine vagal nerve stimulation would help us understand its potential utility for cognitive impairment in people with epilepsy.

\section{Cholinergic replacement}

In animal and human studies, the cholinergic system is the neurotransmitter system traditionally most associated with memory processes. Cholinergic replacement is a focus of Alzheimer's disease therapy. However, the theoretical basis for cholinergic replacement is less direct when memory disorders result from epilepsy. In animals, seizure-induced memory impairment is reduced by choline supplementation before or after status epilepticus (Shulman 2002). A single open-label pilot study of donepezil suggests improvement in certain aspects of memory in memory-impaired individuals with partial (focal) epilepsy (Fisher 2001). In the 3-month trial, patients given 5-10 mg of donepezil could recall a significantly greater number of words on a list-learning task. However, so far there is no evidence from randomised controlled trials in this area. It must also be taken into account that seizures are a potential sideeffect of acetylcholinesterase inhibitors, especially in people with epilepsy. In the open-label donepezil study (Fisher 2001), seizures worsened in 2 of the 18 patients, but there were no statistically significant changes in seizure frequency.

\section{Antidepressants and anxiolytics}

Memory problems associated with anxiety and depression in epilepsy warrant psychopharmacological intervention for these indications. In general, antidepressants with strong anticholinergic effects or sedative actions have a global negative effect on attention, memory and psychomotor speed. Serotonin reuptake inhibitors are the first-line treatment for depression in epilepsy. They have low epileptogenicity, are safer in overdose and cause few deleterious cognitive effects. Citalopram and sertraline, for example, are associated with enhanced psychomotor responses, sustained attention and greater verbal fluency (Shulman 2002).

\section{Stimulants}

Attentional dysfunction, slowed cognition and sedation are frequently encountered in people with epilepsy. Stimulants such as methylphenidate have been studied in patients with intractable seizures who suffer from excessive sedation. An openlabel, non-randomised 3-month study using the drug showed clinically significant improvements in attention/concentration and memory (Moore 2002). The newer stimulant compound modafinil works by altering the hypocretin-orexin system in the hypothalamus. It is approved to treat excessive daytime sleepiness associated with narcolepsy, but there have as yet been no trials of its use in epilepsy. Both methylphenidate and modafinil may prove to be a useful adjunct for treating cognitive dysfunction in epilepsy in the future, but they cannot be clinically recommended at present.

\section{Memory rehabilitation}

Memory rehabilitation strategies can be of immense help to patients with epilepsy who have significant cognitive difficulties. These consist of any strategy or technique intended to enable individuals - and their families - to live with, manage, bypass, reduce or come to terms with cognitive deficits precipitated by injury to the brain. General aspects of memory rehabilitation

\section{B0X 4 General aspects of memory rehabilitation}

\section{Psychoeducation}

The first important step is creating realistic perspectives about the impact and possibilities for improvement in memory problems

Personality changes and emotional reactions These must be taken into account before treatment starts, as they can interfere with the rehabilitation process

Individual perception of cognitive disorders

A discrepancy between the severity of objective memory impairment and subjective memory complaints may be attributable to a loss of memory confidence: this should be addressed 


\section{BOX 5 Compensatory memory strategies}

\section{External strategies}

Storing information externally, e.g. calendar, agenda, diary

Reminders to perform specific activities in the future, e.g. watch alarm, personal digital assistant

Rearrangement of environment, e.g. grouping of all the things needed for breakfast on one shelf in the kitchen

Internal strategies

Verbal strategies, e.g. PQRST method to remember text:

Preview: first reading

Questions: make questions about the content

Read: read again and answer the questions

State: repeat the content

Test: answer the questions again

Visual imagery strategies

Face-name associations, etc

programmes are summarised in Box 4. The restoration of cognitive function is not a goal: the keyword is compensation. The patient learns alternative ways to cope with the daily flow of information (e.g. remembering) instead of trying to enhance information processing capacities (e.g. memory in general) (Ponds 2006).

The specific aspects of such programmes are the memory problems that should be trained and the strategies that should be used. Thus, training goals are tailor made, small, concrete, realistic and adjusted to the needs and wishes of the patient. One compensatory approach adopted in memory rehabilitation involves patients learning to use specific mnemonic strategies to remedy specific everyday problems. Compensatory memory strategies are divided into two categories: external and internal (Box 5) (Ponds 2006).

Some individuals have problems successfully retrieving information that is already stored in memory. One way to overcome this is to teach the individual to perform systematic searches in the handicapped memory system, focusing on what, where, who, when - the four Ws - when trying to remember specific events (Ponds 2006).

\section{Conclusions}

All psychiatrists will come across patients with epilepsy in their clinical practice. They may present with psychiatric problems such as depression, anxiety or psychosis, and they may also be affected by cognitive impairments. It is clear from the literature that epilepsy-related cognitive impairment arises from complex interactions of multiple interrelated factors. In the majority of patients, the key factors are the aetiology of seizures, seizure type, seizure frequency, the sideeffects of anti-epileptics on the central nervous system and comorbid psychiatric conditions such as anxiety and depression. Management of memory problems in epilepsy involves effective control of seizures using anti-epileptics that are less likely to affect cognitive function, treating comorbid psychiatric illness and using memory rehabilitation strategies. Other possible direct treatments currently remain experimental and cannot be recommended for clinical practice.

\section{References}

Airaksinen E, Larsson M, Forsell Y (2005) Neuropsychological functions in anxiety disorders in population-based samples: evidence of episodic memory dysfunction. Journal of Psychiatric Research 39: 207-14.

Aldenkamp AP (2001a) Effects of antiepileptic drugs on cognition. Epilepsia 42 (suppl 1): 46-9

Aldenkamp AP, Baker G (2001b) A systematic review of the effects of lamotrigine on cognitive function and quality of life. Epilepsy \& Behavior 2: 85-91.

Aldenkamp AP, Bodde N (2005) Behaviour, cognition and epilepsy. Acta Neurologica Scandinavica 112 (suppl 182): 19-25.

Aldenkamp AP (2006) Cognitive impairment in epilepsy: state of affairs and clinical relevance. Seizure 15: 219-20.

Baxendale $S$ (2008) The impact of epilepsy surgery on cognition and behaviour. Epilepsy \& Behavior 12: 592-9.

Blake RV, Wroe SJ, Breen EK, et al (2000) Accelerated forgetting in patients with epilepsy: evidence for an impairment in memory consolidation. Brain 123: 472-83.

Brodie M, McPhail E, Macphee G, et al (1987) Psychomotor impairment and anticonvulsant therapy in adult epileptic patients. European Journal of Clinical Pharmacology 31: 655-60

Butler C, Graham K, Hodges J, et al (2007) The syndrome of transient epileptic amnesia. Annals of Neurology 61: 587-98.

Butler CR, Zeman A (2008) Recent insights into the impairment of memory in epilepsy: a systematic review of transient epileptic amnesia, accelerated long-term forgetting and remote memory impairment. Brain 131: 2243-63.

Butler C, Bhaduri A, Acosta-Cabronero J, et al (2009) Transient epileptic amnesia: regional brain atrophy and its relationship to memory deficits. Brain 132: 357-68.

Dodrill C (1986) Correlates of generalised tonic-clonic seizures with intellectual, neuropsychological, emotional and social function in patients with epilepsy. Epilepsia 27: 399-411.

Elger C, Helmstaedter C, Kurthen M (2004) Chronic epilepsy and cognition. Lancet Neurology 3: 663-72.

Fisher R, Bortz J, Blum D, et al (2001) A pilot study of donepezil for memory problems in epilepsy. Epilepsy \& Behavior 2: 330-4.

Gleissner U, Helmstaedter C, Elger C (2002) Memory reorganisation in adult brain: observations in three patients with temporal lobe epilepsy. Epilepsy Research 48: 229-34.

Gualtieri C, Johnson L, Benedict K (2006) Neurocognition in depression: patients on and off medication versus healthy controlled subjects. Journal of Neuropsychiatry and Clinical Neurosciences 18: 217-25.

Helmstaedter C, Elger C (1999) The phantom of the progressive dementia in epilepsy. Lancet 354: 2133-4.

Helmstaedter C, Brosch T, Kurthen M, et al (2004) The impact of sex and language dominance on material-specific memory before and after left temporal lobe epilepsy. Brain 127: 1-8. 
Hirsch E, Schmitz B, Carreno M (2003) Epilepsy, antiepileptic drugs (AEDs) and cognition. Acta Neurologica Scandinavica 108 (suppl 180): 23-32.

Hommet C, Hannelore S, De Toffel B, et al (2006) Idiopathic epilepsy syndromes and cognition. Neuroscience and Biobehavioural Reviews 30 : 85-96.

Lagae $L$ (2006) Cognitive side-effects of anti-epileptic drugs: the relevance in childhood epilepsy. Seizure 15: 235-41.

Langfitt JT, Rausch R (1996) Word-finding deficits persist after left anterotemporal lobectomy. Archives of Neurology 53: 72-6.

Leijten FS, Alpherts WC, Van Huffelen AC, et al (2005) The effects on cognitive performance of tailored resection in surgery for nonlesional mesiotemporal lobe epilepsy. Epilepsia 46: 431-9.

Lezak M, Howieson D, Loring D (2004) Neuropsychological Assessment. Oxford University Press.

Mameniskiene R, Jatuzis, Kaubrys G, et al (2006) The decay of memory between delayed and long-term recall in patients with temporal lobe epilepsy. Epilepsy \& Behavior 8: 278-88.

McCagh J, Fisk J, Baker G (2009) Epilepsy, psychosocial and cognitive functioning. Epilepsy Research 86: 1-14.
Moore J, McAuley J, Long L, et al (2002) An evaluation of the effects of methylphenidate in adult epilepsy patients. Epilepsy \& Behavior 3: 92-5.

Nordli D (2005) Idiopathic generalized epilepsies recognized by the International League Against Epilepsy. Epilepsia 46 (suppl 9): 48-56.

Ponds R, Hendriks M (2006) Cognitive rehabilitation of memory problems in patients with epilepsy. Seizure 15: 267-73.

Privitera M, Fincham R, Penry J, et al (1996) Topiramate placebocontrolled dose-ranging trial in refractory partial epilepsy using 600800-, and 1,000-mg daily dosages. Neurology 46: 1678-83.

Sanyal SK, Chandra PS, Gupta S, et al (2005) Memory and intelligence outcome following surgery for intractable temporal lobe epilepsy: relationship to seizure outcome and evaluation using a customized neuropsychological battery. Epilepsy \& Behavior 6: 147-55

Shulman M, Barr W (2002) Treatment of memory disorders in epilepsy. Epilepsy \& Behavior 3 (suppl 5): 30-4.

Zeman A, Boniface S, Hodges R (1998) Transient epileptic amnesia: a description of the clinical and neuropsychological features in 10 cases and a review of literature. Journal of Neurology, Neurosurgery \& Psychiatry 64: 435-43.

\section{MCOs}

Select the single best option for each question stem

1 Juvenile myoclonic seizures:

a are a type of idiopathic partial epilepsy

b account for $30 \%$ of all epilepsies in adolescents and young adults

c occur between the ages of 3 and 13 years

$\mathrm{d}$ are also known as Janz syndrome

e are not associated with dysexecutive syndrome.

\section{Transient epileptic amnesia:}

$a$ is a subtype of frontal lobe epilepsy

b presents mainly as tonic-clonic seizures

c is associated with deficits in executive functions

d tends to last for more than an hour

e may be associated with interictal autobiographical amnesia
3 Anti-epileptic drugs:

a affect cognitive function by increasing neuronal activity

b all cause severe cognitive side-effects

c of the newer type have a less favourable cognitive profile than older anti-epileptics

d affect neuropsychological functions such as psychomotor speed, vigilance, memory and language functions

e such as topiramate, when used as adjunctive therapy in intractable epilepsy, lead to significant improvement in verbal 10 , verbal fluency and verbal learning.

\section{Epilepsy surgery:}

a is associated with up to $35 \%$ post-operative improvement in memory

b that involves temporal lobe resection leads to non-verbal memory deficits

c such as selective amygdala-hippocampectomy may be associated with fewer post-operative cognitive changes than a standardised en bloc temporal resection

$\mathrm{d}$ always leads to an increase in the use of antiepileptics

e never affects mood post-operatively.

5 Management of memory problems in epilepsy:

a is more successfully achieved by indirect methods and a rehabilitative approach than by direct methods such as pharmacotherapy

b involves direct methods such as anti-epileptics

c includes the treatment of depression with tricyclic antidepressants as the first-line drug

$\mathrm{d}$ using verbal strategies in memory rehabilitation is not successful

e involves the use of stimulants such as modafinil, which work on the hypocretin-orexin system in the thalamus 